\title{
Power Consumption Modeling and Analysis of Integrated Optical-Wireless Access Network
}

\author{
A. Ramli ${ }^{1}$, N. Zulkifli ${ }^{2}$, S. M. Idrus ${ }^{3}$ \\ Lightwave Communication Research Group (LCRG), Fakulti Kejuruteraan Elektrik, \\ Universiti Teknologi Malaysia, Johor, Malaysia
}

\begin{tabular}{l} 
Article Info \\
\hline Article history: \\
Received Aug 29, 2016 \\
Revised May 28, 2017 \\
Accepted Jun 20, 2017 \\
\hline
\end{tabular}

\section{Keyword:}

Optical access

Wireless access

GPON

Femtocell

Power consumption model

\begin{abstract}
The integration of optical and wireless technologies at access networks are considered as a future solution which provide both high bandwidth and high mobility in an efficient way. GPON is a suitable candidate for optical backhaul due to the combination of higher data rates, greater split ratio and support for triple play services hence it offers maximum flexibility and cost advantages. On the other hand, recent developments of new radio access technologies and introduction of femtocell base stations provide the potential of offering broadband services and applications to everyone and everywhere. However, the power consumption of this network demands a particular attention because access networks are the largest contributor the network related electricity consumption. Therefore, in this paper we evaluate the power consumption of integrated optical-wireless access network which is based on independent ONU-BS architecture. We proposed a power consumption model for such network and the assessment has been done under different simulation scenarios. The constructed model will provide insight of the energy performance of the integrated access network so that in the network design process, focus can be done to the most energy saving strategies.
\end{abstract}

Copyright $\odot 2017$ Institute of Advanced Engineering and Science. All rights reserved.

\section{Corresponding Author:}

A. Ramli,

Lightwave Communication Research Group (LCRG),

Fakulti Kejuruteraan Elektrik,

Universiti Teknologi Malaysia, 81310 UTM Skudai, Johor, Malaysia.

Email: arnidza@ fke.utm.my

\section{INTRODUCTION}

Research on passive optical network $(\mathrm{PON})$ continues at a remarkable pace where the future solution for PON with extended data rates in order to meet broadband consumer demand was recommended [1],[2]. The planning was divided into two phases; NG-PON1 and NG-PON2. NG-PON1 is expected to deliver data rates up to $10 \mathrm{Gbps}$ based on the existing Gigabit-capable PON (GPON) legacy whereas NGPON2 include the research area of time and wavelength division multiplexed PON (TWDM-PON) which is capable to provide data rates no less than $40 \mathrm{Gbps}$. In addition to the high bandwidth demand, increasing mobility requirements for access networks also present new challenges for service providers. Mobility is highly desirable for users, because it enables access to the Internet regardless of location. According to surveys, mobile data traffic has grown 4000 -fold over the past 10 years and will be increased nearly eightfold between 2015 and 2020 [3]. Moreover, as the Internet of Things (IoT) becomes a reality, there will be massive growth in the number of connected devices which is expected to be around 12 billion devices by 2020.

The integration of optical and wireless networks is a good alternative to overcome both issues to the complementary features of wide bandwidth and user mobility in optical and wireless networks, respectively. However, due to the expansion of network connectivity and the increment of data rate of the network, the 
power consumption is expected to increase. The optical access network consumes $60-80 \%$ of the total power consumed by a wired network [4],[5] where the power consumption is dominated by the Optical Network Units (ONUs). On the other hand, wireless access network consumes $9 \%$ of ICT power consumption [6] where $80 \%$ of the power is consumed by the Base Stations (BSs) [7]. Therefore, the study on the energy consumption of such network will allow energy performance optimization, network architecture improvement and other network parameters enhancement to be applied. Thus, the power consumption and energy efficiency of the integrated access network become a desirable objective in the current research direction.

\subsection{Integrated Optical-Wireless Access Network}

Optical access networks are being widely deployed nowadays where it can support high capacity and longer distance communication. PON is the most widely deployed optical access technology. To date, several PON standards have been proposed in order to improve the performance of PON technologies such as EPON and 10G-EPON standardized by IEEE and GPON and NG-PON by ITU-T. Table 1 provides summary of the enabling PON technologies. On the other hand, wireless access networks have evolved from the first generation $(1 \mathrm{G})$ until the fifth generation $(5 \mathrm{G})$, improving the transfer speed and including new technologies to provide service for new packet formats, applications and requirements of users. Currently, we are at the 4G which refers to all-IP packet-switched networks, mobile ultra-broadband (gigabit speed) access and multicarrier transmission. Long Term Evolution (LTE) and Worldwide Interoperability for Microwave Access (WiMAX) is a version of $4 \mathrm{G}$ which promise data rates of $100 \mathrm{Mbps}$. Table 2 summarized the characteristics of these wireless generations. Apart from access technology evolutions in order to satisfy increasing bandwidth demand, other trends to emerge are femtocells. Femtocells are small, low power BS typically design for use in a home or small business. It can significantly boost up wireless network capacity [8] and studies in [9] has shown that the deployment of femtocells can significantly increase both access data rates and energy efficiency in areas with high population densities thus making the femtocell as an attractive solution in order to increase network capacity and coverage in an economical way.

Table 1. Characteristics of different optical access technologies

\begin{tabular}{|c|c|c|c|c|c|c|}
\hline & \multicolumn{2}{|c|}{ Data rate (Gbps) } & \multicolumn{2}{|c|}{ Wavelength (nm) } & \multirow{2}{*}{$\begin{array}{l}\text { Typical Max. } \\
\text { Splitting ratio }\end{array}$} & \multirow{2}{*}{$\begin{array}{l}\text { Typical Max. } \\
\text { Distance }(\mathrm{km})\end{array}$} \\
\hline & DS & US & DS & US & & \\
\hline GPON & 2.44 & 1.24 & 1490 & 1310 & $1: 64$ & 20 \\
\hline EPON & 1.25 & 1.25 & 1490 & 1310 & $1: 16$ & 20 \\
\hline \multirow[t]{2}{*}{ 10G EPON } & 10 & 10 & $1575-1580$ & $1260-1280$ & $1: 16$ & 20 \\
\hline & 10 & 1 & & & $1: 32$ & \\
\hline NG-PON1 & 10 & 2.5 & $1575-1580$ & $1260-1280$ & $1: 128$ & 20 \\
\hline NG-PON 2 & 40 & 40 & \multicolumn{2}{|c|}{ N/A } & $1: 1024$ & 100 \\
\hline
\end{tabular}

Table 2. Generation in wireless access technologies

\begin{tabular}{|c|c|c|c|c|c|}
\hline & Year Introduced & Technology & Multiple Access System & Data Rates & Operating frequencies \\
\hline $1 \mathrm{G}$ & $1980 \mathrm{~s}$ & AMPS, NMT, TACS & FDMA & $2.4 \mathrm{kbps}$ & $800 \mathrm{MHz}$ \\
\hline $2 \mathrm{G}$ & 1993 & GSM, GPRS, EDGE & TDMA, CDMA & $64 \mathrm{kbps}$ & $900 \mathrm{MHz}, 1800 \mathrm{MHz}$ \\
\hline $3 \mathrm{G}$ & 2001 & UMTS, HSPA & CDMA & 3.1 Mbps & $2100 \mathrm{MHz}$ \\
\hline $4 G$ & 2009 & LTE, WiMAX & CDMA & $100 \mathrm{Mbps}$ & $850 \mathrm{MHz}, 1800 \mathrm{MHz}$ \\
\hline $5 \mathrm{G}$ & 2020 (expected) & Beyond LTE & CDMA & $1-10$ Gbps & Sub-GHz range \\
\hline
\end{tabular}

Trends have shown that traffic from wireless and mobile devices will surpass traffic from wired devices by year 2018 [10]. Moreover, future broadband access networks not only have to provide access to information anywhere, anytime and in whatever format we need it, but also more importantly have to bridge the digital divide and offer simplicity and user-friendliness in order to stimulate the design of new applications and services. To achieve this goal, optical and wireless access networks can be integrated in a complementary way. Some works have been focused on this integrated network are reported in [11],[12]. According to [12], there are potentially four integrated architectures; namely the independent ONU-BS, hybrid ONU-BS, unified connection-oriented and microwave over fiber. Independent ONU-BS directly connects ONU to the BS through standard Ethernet interface. Contrary, hybrid ONU-BS integrates ONU and BS in both hardware and software. Therefore, modification on the ONU/BS architecture is required. Unified connection-oriented deploys similar architecture as hybrid ONU-BS. However, the unified architecture can be run like a WiMAX network with the ability to grant bandwidth finely using WiMAX's connectionoriented. On the other hand, microwave over fiber modulate WiMAX signal on a wireless carrier frequency 
and then is multiplexed and modulated together with the baseband optical signal onto a common optical frequency at the ONU-BS. In this work, we consider the independent ONU-BS architecture because the ONU and BS acts independently thus no modification on the ONU and BS is required. Consequently, the energy modeling can be done separately for both ONU and BS providing ease of modeling development.

\subsection{Related Work}

To date numerous studies have been done in the field of integrated optical wireless technologies in order to meet the cost challenges and provides ubiquitous broadband connections for the next generation broadband access network. The integration work has been focused on i) physical layer, ii) MAC layer and iii) network layer. In [13], the authors proposed hybrid BS scheme based on reflective semiconductor optical amplifier (RSOA) that enables both wireless and wired services together by making it laser free. However, the scheme is relatively expensive since the optical carrier is required to be delivered to each hybrid BS. Thus, they later proposed and experimentally demonstrate hybrid BS architecture that uses self seeding of the RSOA [14]. As a result, a secondary feeder fiber is not required and therefore reducing the cost of deployment. In other hand, authors in [15] have proposed a similar hybrid BS architecture as previous but instead of using RSOA, they used vertical cavity surface emitting laser (VCSEL) as the optical source which further provides cost effectiveness without any temperature control.

Authors in [16] have presented a dynamic scheduling algorithm namely up-link scheduler with the purpose of improving upstream bandwidth utilization for WDM EPON. In [17], the authors have proposed a centralized scheduling mechanism that shortens the packet delay and the time of transmitting data upon requesting bandwidth. Meanwhile, the work on energy efficiency of the integrated access network can be found in [9],[18],[19]. In [18], the authors discussed the energy efficient bandwidth allocation mechanism that adopts an optical burst switching technique for long reach network. Meanwhile, authors in [19] have reported the sleep mode algorithm for energy efficient FiWi networks. In [9], the authors presented the energy efficiency analysis for the integrated optical wireless access networks deploying femtocells. To the best of our knowledge, research on energy efficiency of the integrated access networks generally focus on Layer 2 and 3 solutions. Unfortunately, the power consumption study on the integration of wireless networks with the existing fiber based PON remains significantly unexplored. Eventhough analysis of power consumption for such networks have been evaluated in [10], the power consumption model for the network elements is derived based only on the values provided in the datasheets. These values are often oversimplified and do not represents the real consumption. Therefore, in this paper we provide the detailed modeling of the power consumption of the network elements where we considered the power consumption dependence on the traffic load and site factors.

\section{RESEARCH METHOD}

The architecture of the considered access network is shown in Figure 1. The wireless access is optically backhaul where we considered GPON for optical network and femtocell base station (FBS) for wireless network. In this integrated architecture, the ONUs and FBSs are connected via common Ethernet interfaces. Such integrated architecture is known as Independent ONU-BS (IOB). We predicted that in the future demand for high mobility will be increased since it enables access to the Internet anytime and anywhere. In addition, surveys have projected that there will be 11.6 billion mobile-connected devices by 2020 [3]. Hence, we assume all ONUs within the network are connected with the FBSs.

In our modeling approach, the total power consumption of this integrated network is the sum of contribution from OLTs and IOBs connected within the network. We set our system boundaries at network terminals thus the power consumption of end-user equipment such as personal computers (PCs), laptops and smartphones is not taken into account. In addition, mobile devices consume just a small fraction of the energy consumed by optical and wireless access network element since they are optimized for low energy consumption. Therefore, the total power consumption of the integrated access network is:

$$
P_{I O W}=\sum_{i=1}^{M} P_{i}^{O L T}+\sum_{i=1}^{N} P_{i}^{I O B}
$$

in which the power consumption of IOB $i$ is expressed as follows:

$$
P_{i}^{I O B}=P_{i}^{O N U}+P_{i}^{F B S}
$$

where $M$ and $N$ are the number of OLT and IOB respectively, and $P_{i}^{O L T}, P_{i}^{I O B}, P_{i}^{O N U}$ and $P_{i}^{F B S}$ are the power consumption of OLT, IOB, ONU and FBS respectively. $M$ is determined by using this equation: 


$$
M=\left\lceil\frac{N}{S R}\right\rceil
$$

where SR is the splitting ratio of the passive splitter.

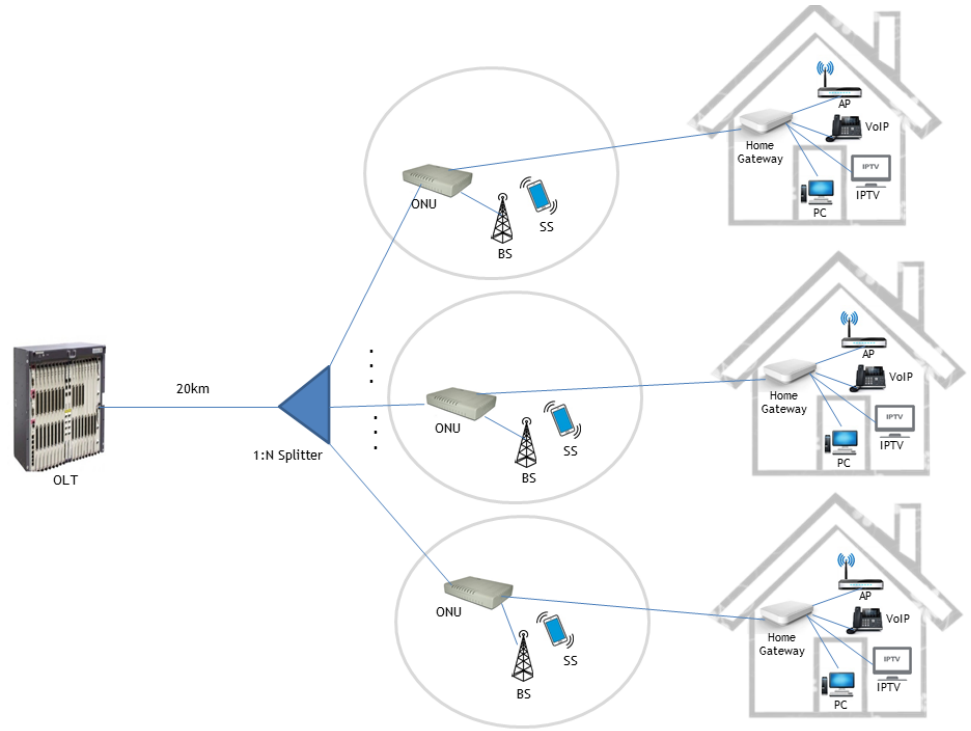

Figure 1. Integrated optical and wireless access network

\subsection{Power consumption model of OLT}

The power consumption of OLT is assumed independent of traffic load. Instead, it is influenced more by the ambient temperature, electronic components and power conversion efficiency which will be taken into account in our modeling later. In addition, it is challenging to put OLT into sleep mode since it might cause a synchronization problem for the ONUs [20]. Therefore, we consider the power consumption of OLT is fixed hence the power consumption of the OLT is modeled based on its functional blocks and the components that consume power was identified. The generic structure of the OLT is depicted in Figure 2.

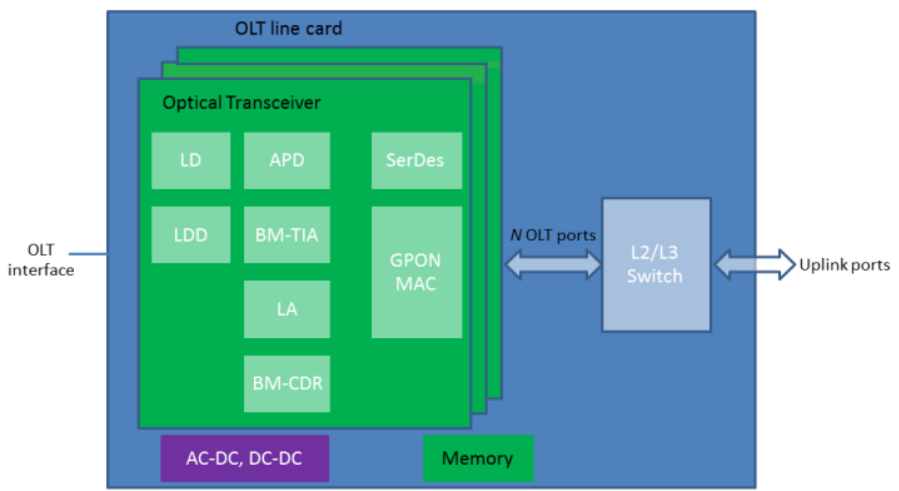

Figure 2. Generic structure of OLT

The OLT element usually comprises an uplink port, a switching module and a number of OLT line cards. Therefore, the power consumption of OLT is the sum of the contributions from OLT PON ports, general OLT functions such as L2/L3 switching, packet processing and traffic management and uplink ports. Power conversion efficiency and site factors such as AC/DC rectifiers, ventilation and air conditioning, auxiliary power units and batteries are also considered in this modeling approach. For the OLT $i$, the power consumption model is expressed as follows: 


$$
P_{i}^{O L T}=\left(P_{\text {ports }}+P_{\text {control }}+P_{U L}\right) \times \frac{1}{\eta_{D C / D C}} \times S F
$$

where $P_{\text {ports }}, P_{\text {control }}$ and $P_{U L}$ are power consumption of OLT PON ports, general OLT function and uplink ports respectively, $\eta_{D C / D C}$ is power conversion efficiency and $S F$ is site factor.

\subsection{Power consumption model of $\mathrm{ONU}$}

Figure 3 illustrated the generic structure of the ONU. The network model element for the ONU is divided into three modules which are represented by different color codes; Optical transceiver, System on Chip (SoC)/signal processing and Interfaces. The power consumption of the ONU was modeled based on its dependence on the traffic loads. The power consumption of the ONU was experimentally measured by using real GPON testbed in which we utilized Arduino-based energy meter for power monitoring and measurement. Based on the obtained results, we model the power consumption of ONU $i$ as:

$$
P_{i}^{O N U}=\alpha_{o} r_{o}+\gamma_{o}
$$

where $\alpha_{o}$ is the power consumed by the ONU to transmit or receive 1 bit of information, $r_{o}$ is the average access data rate per $\mathrm{ONU}$ and $\gamma_{o}$ is the power consumption of ONU when idle.

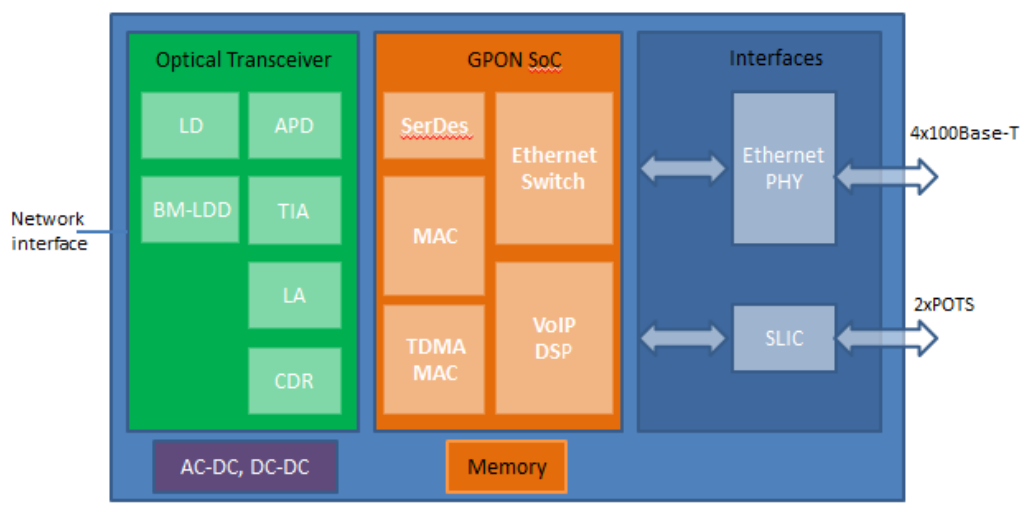

Figure 3. The generic structre of ONU

\subsection{Power consumption model of femtocell base station (FBS)}

The FBS's generic structure can be found in Figure 4. We consider femtocell BSs because it is the most energy efficient compared to other type of BSs [9]. Similarly to ONU, the BS can be classified into three basic modules; RF transceiver, DSP/signal processing and interfaces. The power consumption model for FBS used in this study is based on the work previously reported in [21]. They used similar approach as our modeling for the ONU which is the power consumption for the FBS is measured by using an Arduinobased energy meter. For the FBS $i$, the power consumption model is as follows:

$$
P_{i}^{F B S}=\alpha(d) r_{w}+\beta(d)+\gamma_{w}
$$

where $r_{w}$ is the average data rate of wireless users, $\beta(d)$ is the dependence of power consumption on datagram length and $\gamma_{w}$ is the power consumption when FBS is idle. $\alpha(d)$ is given by the equation:

$$
\alpha(d)=\alpha\left(1+\frac{\alpha_{1}}{d}\right)
$$

where $\alpha_{0}$ is the factor converting between units of bytes and energy, $\alpha_{1}$ is the per datagram overhead and $d$ is the datagram size in Bytes. 


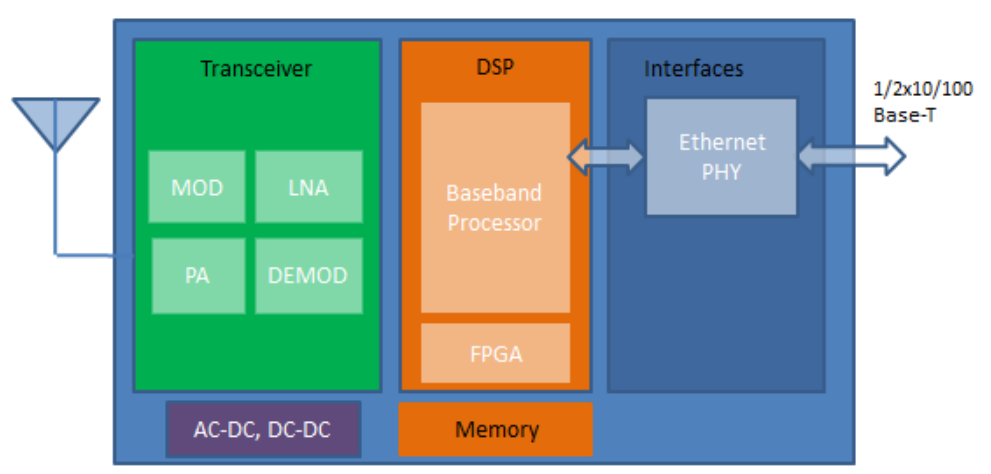

Figure 4. The generic structure of FBS

\subsection{Considered scenarios}

This section describes the assumptions made and the scenarios considered in this study. Several deployment scenarios are defined for a fixed network coverage area and different FBS range. The FBS is a small cell which typically has coverage radius less than $50 \mathrm{~m}$. From the value of cell coverage radius or range $R$, we can determine the cell coverage area $A$ by using equation (8) [22]:

$$
A=\frac{3}{2} \sqrt{3} R^{2}
$$

In our simulation scenario, we assume coverage area of $100 \mathrm{~km}^{2}$ and vary the FBS range from $10 \mathrm{~m}$ to $50 \mathrm{~m}$. These parameters were then used to determine the number of IOBs $N$ :

$$
N=\left\lceil\frac{S}{A}\right\rceil
$$

where $S$ is the network coverage area and $A$ is the cell coverage area. Additionally, we assumed that there is no more than one active user within a femtocell at a time.

Other parameters considered in the simulation are shown in Table 3. We assume datagram size of $1470 \mathrm{~B}$. The average access data rates for wired users is assume at $10 \mathrm{Mbps}$ based on the typical data rate subscribe by the residential users. In other hand, the average data rates for wireless users is based on the data provided in [3] which is $3.1 \mathrm{Mbps}$ for the year of 2016.

\begin{tabular}{|c|c|c|c|c|c|}
\hline \multicolumn{2}{|c|}{ OLT } & \multicolumn{2}{|c|}{ ONU } & \multicolumn{2}{|c|}{ FBS } \\
\hline Parameter & Value/Unit & Parameter & Value/Unit & Parameter & Value/Unit \\
\hline $\boldsymbol{P}_{\text {ports }}$ & $41.5 \mathrm{~W}$ & $\gamma_{o}$ & $11.53 \mathrm{~W}$ & $\alpha$ & $0.06 \mathrm{~W} / \mathrm{Mbps}$ \\
\hline$P_{\text {control }}$ & $33.3 \mathrm{~W}$ & $\alpha_{o}$ & $0.00237 \mathrm{~W} / \mathrm{Mbps}$ & $\alpha_{1}$ & $70 \mathrm{~B}$ \\
\hline $\boldsymbol{P}_{U L}$ & $2.3 \mathrm{~W}$ & $r_{o}$ & $10 \mathrm{Mbps}$ & $d$ & $1470 \mathrm{~B}$ \\
\hline$\eta_{D C / D C}$ & 0.9 & & & $r_{w}$ & 3.1 Mbps \\
\hline$S F$ & 1.7 & & & $\beta(d)$ & $0.27 \mathrm{~W}$ \\
\hline & & & & $\gamma_{w}$ & $7.77 \mathrm{~W}$ \\
\hline
\end{tabular}

Table 3. Parameters considered in the simulation [21],[23],[24]

We also considered the effect of power consumption performance when the network is connected to different wireless access technologies. We take into account the wireless technologies that applicable for small cell application such as UMTS, HSPA, WiMAX and LTE.

\section{RESULTS AND ANALYSIS}

The simulation results are presented in this section. The power consumption model developed and described in the previous section is applied to estimate the total power consumed by the integrated optical wireless access network as shown in Figure 5. The power consumption is plotted versus the coverage area of $100 \mathrm{~km}^{2}$ and the cell range was varied from $10 \mathrm{~m}$ to $50 \mathrm{~m}$ which is in the range of typical coverage radius of femtocell BS. 


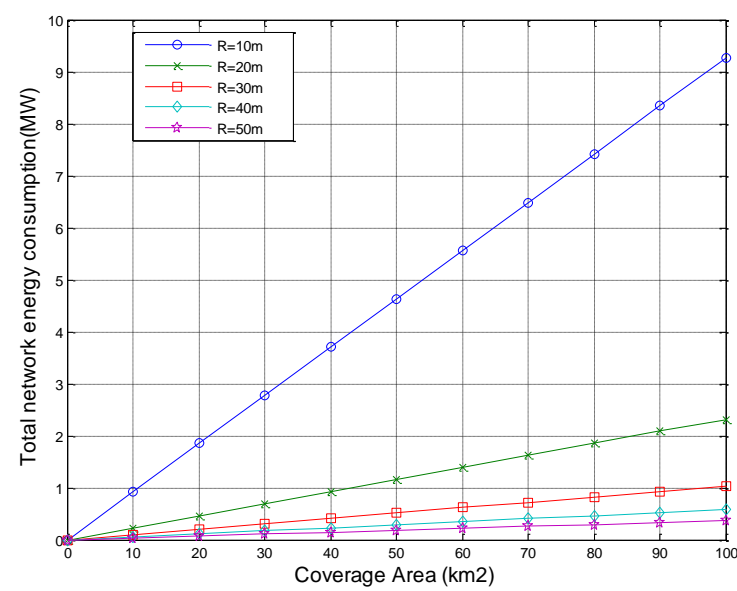

Figure 5. Total network's power consumption with different FBS range

Figure 5 shows the total network power consumption of the integrated optical-wireless access network with different femtocell BS range versus coverage area from 0 to $100 \mathrm{~km}^{2}$. The difference on the cell range has result in the different coverage area of the femtocell BS as refers to Equation 7. Consequently, the number of IOBs connected to the OLT will also be affected depending on the area that the FBS is able to cover.

As can be seen in Figure 5, a deployment of wider cell range leads to decreased total network power consumption. This is due to the fact that lower number of FBSs are needed to cover the considered area. Since the power consumption is proportional to the number of network elements thus, less number of FBSs can be achieved by deploying high coverage range resulting in the lowest value of total network power consumption. It can also be found that the difference in the power consumption from the highest consumption to the lowest consumption is also decreased in which for coverage area of $100 \mathrm{~km}^{2}$, the highest difference is $75 \%$ and reduced to around $36 \%$ as the range increases. Thus, it is better to utilize the optimum range which can provide better coverage with low power consumption.

In our simulation, we also considered the implementation of different wireless access technologies to the GPON backhaul of the integrated access network. We evaluated its effect to the power consumption performance of such network. The simulation result is presented in Figure 6 in which we applied several wireless access technologies that represent the different wireless generation. Since the developed power consumption of the integrated network is suitable for small cell application, therefore we only consider the technologies from $3 \mathrm{G}$ to $4 \mathrm{G}$ which is applicable to the femtocell BS. From the previous results, it can be seen that the FBS range of $50 \mathrm{~m}$ consumed the least power. However, the wider range of femtocell BS might reduces the signal strength [25],[26]. Therefore, in this simulation we take into account the femtocell range of $30 \mathrm{~m}$ which offers acceptable amount of power consumption with better network coverage.

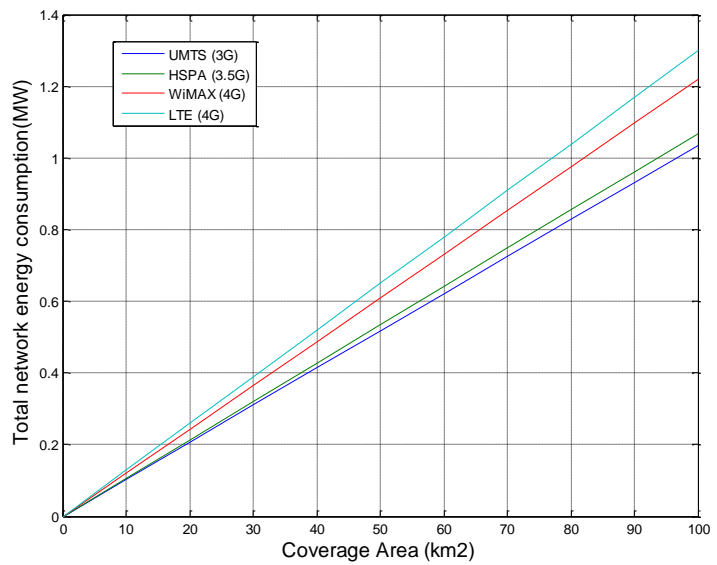

Figure 6. Total network power consumption with different wireless access technologies 
Figure 6 depicted the total network power consumption of integrated GPON with different wireless technologies i.e UMTS, HSPA, WiMAX and LTE versus coverage area of 0 to $100 \mathrm{~km}^{2}$. Note that all parameters are common for all four wireless technologies. However, the parameter $r_{w}$ differs where it is set at $2 \mathrm{Mbps}, 14.4 \mathrm{Mbps}, 75 \mathrm{Mbps}$ and $100 \mathrm{Mbps}$ for UMTS, HSPA, WiMAX and LTE respectively. The differences are due to the fact of the difference peak data rate offer by these technologies in accordance to which wireless generation they are in.

By referring to Figure 6, it was found that higher wireless generation connected to the GPON backhaul resulting in the higher power consumption of the network. The wireless technologies have evolved from $1 \mathrm{G}$ to $4 \mathrm{G}$ and in the future to $5 \mathrm{G}$ in order to satisfy user demand for high bandwidth and high speed services. Thus, the data rates offer by these technologies increased in accordance to the higher wireless generation. As a result, the power consumption of such technologies connected to the network is increased. Unfortunately, the power consumption is expected to further increase due to the growing demand for broadband services in the future. As a result, the implementation of power saving technique to the broadband access network is significantly essential towards greener network environment.

\section{CONCLUSION}

In conclusion, we presented a novel model for evaluating the power consumption of the integrated optical wireless access network which is based on independent ONU-BS architecture. Typically, the network elements in optical access network are assumed always on and their consumption is both traffic and time independent. In our model, we take into account the dependence of both ONU and BS to the average traffic. We demonstrate the use of this model to predict the power consumption of the integrated access network particularly for small cell application. It was found that for wider BS range, the power consumption is significantly lower. Additionally, the difference in power consumption becomes smaller where the smallest difference shows a percentage at approximately 36\%. Meanwhile, for the higher wireless generation attached to the GPON backhaul, the higher the power consumption is observed. Although the power consumption is increased, it is worth noted that the data rates offer by the latest technologies is also greater. In other words, these technologies might have potential to offer more energy efficient network. Thus, the future direction of this work will focus on the assessment of energy efficiency and the potential of energy saving technique to this integrated access network.

\section{ACKNOWLEDGEMENTS}

This work is supported by Ministry of Education and the administration of Universiti Teknologi Malaysia (UTM) for the project financial support through FRGS Fund vote number 4F407. The authors acknowledge the Universiti Teknologi Malaysia for the financial support through SLAI funding to the first author.

\section{REFERENCES}

[1] J. Zuoqian, "Outlook and overview of PON technology," Huawei Technologies, pp. 51-53, 2008.

[2] F. Effenberger, et al., "An Introduction to PON Technologies," IEEE Communications Magazine: Topics in Optical Communications, pp. 17-25, 2007.

[3] "Cisco Visual Networking Index (VNI) Update Global Mobile Data Traffic Forecast," 2015.

[4] A. Dixit, et al., "ONU power saving modes in next generation optical access networks : progress, efficiency and challenges," Opt. Express, vol/issue: 20(26), 2012.

[5] M. Tadokoro, et al., "Power-saving technologies for network equipment and their application-ONU/wireless-LAN sleep technologies," NTT Technical Review, vol/issue: 12(3), 2014.

[6] M. Deruyck, et al., "Reducing the power consumption in wireless access networks: Overview and recommendations," Prog. Electromagn. Res., vol. 132, pp. 255-274, 2012.

[7] S. N. Shahab, et al., "Assessment of area energy efficiency of LTE macro base stations in different environments," J. Telecommun. Inf. Technol., vol/issue: 2015(1), pp. 59-66, 2015.

[8] A. Acakpovi and D. Kogue, "Inteference Mitigation in Femtocellular Networks," Int. J. Informatics Commun. Technol., vol/issue: 3(2), pp. 2-7, 2014.

[9] S. Aleksic, et al., "Energy Efficiency of Femtocell Deployment in Combined Wireless / Optical Access Networks," Comput. Networks, vol/issue: 57(5), pp. 1217-1233, 2013.

[10] A. Ramli, et al., "Integrated Optical and Wireless Access Networks: from the Energy Consumption Perspective," ARPN J. Eng. Appl. Sci., vol/issue: 10(18), pp. 8485-8490, 2015.

[11] A. Al-sherbaz and I. Lami, "W2BC: A Proposal for a Converged Baseband Implementation of WiMax and WiFi Transceivers," Int. J. Inf. Netw. Secur., vol/issue: 2(1), pp. 91-102, 2013.

[12] G. Shen, et al., "Fixed mobile convergence architectures for broadband access: Integration of EPON and WiMAX," 
IEEE Commun. Mag., vol/issue: 45(8), pp. 44-50, 2007.

[13] N. Nadarajah, et al., "Laser free inter-networking hybrid base stations towards convergence of wireless and wired access networks," Electron. Lett., vol/issue: 43(8), pp. 1-2, 2007.

[14] N. Nadarajah, et al., "Wavelength switchable base station architecture supporting upstream access and local internetworking with a single self-seeded reflective semiconductor optical amplifier," in 2008 IEEE International Meeting on Microwave Photonics jointly held with the 2008 Asia-Pacific Microwave Photonics Conference, MWP2008/APMP2008, pp. 221-224, 2008.

[15] G. Abhishek, et al., "A Proposed inter-networking based Hybrid Base Station Toward Simultaneous wireless and Wired Transport for Converged Access Network," Int. J. Innov. Adv. Comput. Sci., vol. 4, pp. 284-289, 2015.

[16] N. Moradpoor, et al., "Hybrid Optical and Wireless Technology Integrations for Next Generation Broadband Access Networks," in 12th IFIP/IEEE International Symposium on Integrated Network Management and Workshops, pp. 1013-1020, 2011.

[17] B. Jung, et al., "Centralized scheduling mechanism for enhanced end-to-end delay and QoS support in integrated architecture of EPON and WiMAX," J. Light. Technol., vol/issue: 28(16), pp. 2277-2288, 2010.

[18] B. Kantarci and H. T. Mouftah, "Energy Efficiency in the Extended-Reach Fiber-Wireless Access Networks," IEEE Netw., pp. 28-35, 2012.

[19] U. R. Bhatt, et al., "Energy Saving Algorithm in FiWi Network," Int. J. Eng. Technol. Sci. Res., vol/issue: 3(4), pp. 161-167, 2016.

[20] A. R. Dhaini, et al., "Energy Efficiency in TDMA-based Next-Generation Passive Optical Access Networks," IEEE/ACM Trans. Netw., vol/issue: 22(3), pp. 850-863, 2014.

[21] R. Riggio and D. J. Leith, "A measurement-based model of energy consumption in femtocells," in IFIP Wireless Days, pp. 1-5, 2012.

[22] Z. H. Talukder, et al., "Cell coverage evaluation for LTE and WiMAX in wireless communication system," World Appl. Sci. J., vol/issue: 22(10), pp. 1486-1491, 2013.

[23] A. Valenti, et al., "Power consumption measurements of access networks in a wide geographical area test bed and economic perspectives," in Future Network \& Mobile Summit 2012 Conference Proceedings, pp. 1-6, 2012.

[24] S. Lambert, et al., "Energy efficiency analysis of high speed triple-play services in next-generation PON deployments," Elsevier B.V., 2014.

[25] O. Osunade and T. Oguntunde, "Practical Examination of Distance on Channel Capacity in IEEE $802.11 \mathrm{n}$ Wireless Signals," Int. J. Eng. Sci. Innov. Technol., vol/issue: 3(6), pp. 1-8, 2014.

[26] K. Ogunjemilua, et al., "An Investigation into Signal Strength of 802.11n WLAN," in Proceedings of the Fifth Collaborative Research Symposium on Security, E-Learning, Internet and Networking, pp. 191-204, 2009.

\section{BIOGRAPHIES OF AUTHORS}
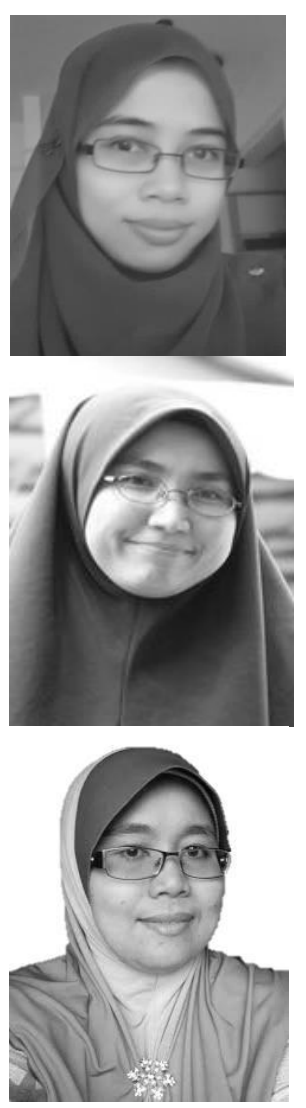

Arnidza Ramli received both the Bachelor degree in Electrical Telecommunication and Master degree in Electrical from the Universiti Teknologi Malaysia in 2007 and 2009 respectively. She is currently a $\mathrm{PhD}$ candidate at the same university. Her current research activity is mainly focused on energy efficiency for the integrated optical and wireless network. Other research interests include power consumption measurement, performance evaluation and green networking.

Nadiatulhuda Zulkifli is a Senior Lecturer in the Department of Communication Engineering, Faculty of Electrical Engineering, Universiti Teknologi Malaysia. She obtained a Bachelor degree in Electrical Telecommunication from UTM and received her masters and PhD degrees from University of Essex, UK. She has authored a number of international journal and conference papers. Her research interests cover optical communication and networking, mobile commerce and innovation adoption.

Sevia Mahdaliza Idrus is the Deputy Director Innovation of the UTM Innovation and Commercilisation Centre and faculty member of the Faculty of Electrical Engineering and Innovative Engineering Research Alliance, UTM. She received her Ph.D in 2004 from the University of Warwick, United Kingdom in optical communication engineering. She has served UTM since 1998 as an academic and administrative staff. Her main research interests are optical communication system and network, optoelectronic design, and engineering management. Her research output have been translated into a number of publications and IPR including a high-end reference books, 'Optical Wireless Communication: IR Connectivity' published by Taylor and Francis, 49 book chapters and monographs, over 150 technical papers, 2 patents granted, 35 patent filings and holds 28 UTM copyrights. 